Revue internationale P.M.E.

Économie et gestion de la petite et moyenne entreprise

\title{
Comparaison et explications des stratégies des PMI françaises et allemandes
}

\section{Jean-Louis Levet et Robert Paturel}

Volume 10, numéro 2, 1997

URI : https://id.erudit.org/iderudit/1009024ar

DOI : https://doi.org/10.7202/1009024ar

Aller au sommaire du numéro

Éditeur(s)

Presses de l’Université du Québec

ISSN

0776-5436 (imprimé)

1918-9699 (numérique)

Découvrir la revue

Citer cet article

Levet, J.-L. \& Paturel, R. (1997). Comparaison et explications des stratégies des PMI françaises et allemandes. Revue internationale P.M.E., 10(2), 81-108.

https://doi.org/10.7202/1009024ar
Résumé de l'article

La France et l'Allemagne sont deux pays européens disposant d'un puissant tissu économique de PME. L'article se propose de rapprocher cette catégorie d'entreprises des deux pays afin de fournir quelques réflexions concernant la refonte de la relation performances des PMI et tissu industriel national. Après quelques précisions méthodologiques, une analyse des comportements stratégiques et des modes de gestion des entreprises industrielles françaises et allemandes de taille moyenne est réalisée afin d'en faire ressortir les dissemblances et les similitudes. Une tentative d'explication des causes structurelles des écarts de performances entre les entreprises françaises et allemandes est ensuite proposée. En conclusion est mise en relief l'idée pour les deux pays, et surtout pour la France, de réarticuler la relation entre les performances des firmes et la compétitivité du système productif national.
Ce document est protégé par la loi sur le droit d'auteur. L'utilisation des services d’Érudit (y compris la reproduction) est assujettie à sa politique d'utilisation que vous pouvez consulter en ligne.

https://apropos.erudit.org/fr/usagers/politique-dutilisation/ 


\title{
Comparaison et explications des stratégies des PMI françaises et allemandes
}

\author{
Jean-Louis LEVET \\ Service du développement technologique et industriel au Commissariat général du plan \\ Robert PATUREL \\ École supérieure des affaires, CERAG
}

\section{MOTS CLÉS}

\section{Alliances - Comportement - Coopération - Flexibilité Impartition - Multicompétence - PME - PMI - Réseau Stratégies génériques de développement - Système productif Territoire - Vraies et fausses PME}

\begin{abstract}
RÉSUMÉ
La France et l'Allemagne sont deux pays européens disposant d'un puissant tissu économique de PME. L'article se propose de rapprocher cette catégorie d'entreprises des deux pays afin de fournir quelques réflexions concernant la
\end{abstract}

\section{LES AUTEURS}

Jean-Louis Levet est directeur du Service du développement technologique et industriel au Commissariat général du plan (France) et professeur associé à l'Université de Poitiers. Détenteur d'un doctorat ès sciences économiques, il a publié de nombreux ouvrages et articles consacrés à l'économie industrielle et à la stratégie d'entreprise. II a, par ailleurs, assumé des responsabilités au sein du ministère de l'Industrie, d'une institution financière, d'un groupe industriel français et auprès du premier ministre Édith Cresson pour les questions industrielles. Adresse : Commissariat général du plan, Service du développement technologique et industriel, 5, rue Casimir-Périer, 75007 Paris, France.

Robert Paturel est professeur à l'Université Pierre-Mendès France de Grenoble, Institut d'études politiques et École supérieure des affaires (France). Il dirige l'équipe de recherche "Stratégies et développement des entreprises" au sein du Centre d'études et de recherches appliquées à la gestion (CERAG) de l'École supérieure des affaires de Grenoble. Il a publié plusieurs dizaines d'articles et ouvrages dans le domaine du management stratégique des organisations. Adresse: École supérieure des affaires, CERAG, B.P. 47, 38040 Grenoble, Cedex 9, France. 
refonte de la relation performances des $P M I$ et tissu industriel national. Après quelques précisions méthodologiques, une analyse des comportements stratégiques et des modes de gestion des entreprises industrielles françaises et allemandes de taille moyenne est réalisée afin d'en faire ressortir les dissemblances et les similitudes. Une tentative d'explication des causes structurelles des écarts de performances entre les entreprises françaises et allemandes est ensuite proposée. En conclusion est mise en relief l'idée pour les deux pays, et surtout pour la France, de réarticuler la relation entre les performances des firmes et la compétitivité du système productif national.

\begin{abstract}
By European standards, both France and Germany have a strong economic fabric of SMEs. The article sets out to place the entrepreneurial features of the two countries side by side in order to reflect on the reform of the relationship between results of SMEs and the nature of the national industrial fabric. A series of methodological considerations are followed by an analysis of the strategic behaviour and management methods of French and German medium-sized industrial enterprises, with a view to highlighting their differences and similarities. This leads on to an attempt to explain the structural causes of the difference in results between French and German firms. In conclusion, the importance of rearticulating the relationship between companies' results and the competitiveness of the national productive system is stressed in connection with both countries, but with France in particular.
\end{abstract}

\title{
RESUMEN
}

Francia y Alemania son dos paises europeos que disponen de un potente tejido economico de PyMEs. El articulo propone acercar estas particularidades de empresas de estos dos paises con el objetivo de proporcionar algunas reflexiones sobre la reforma de la relacion entre los resultados de dichas PyMEs y la naturaleza del tejido industrial nacional. Después de algunas apreciaciones metodologicas, se ha realizado un analisis de los comportamientos estratégicos y de los modos de gestion de las empresas industriales francesas y alemanas de tamano medio, con el objeto de resaltar las diferencias y las similitudes. Una tentativa de explicacion de las causas estructurales de la diferencia de resultados entre las empresas francesas y alemanas se propone a continuacion. En conclusion, se pone en relieve la idea por parte de los dos paises pero sobretodo de parte francesa, de rearticular la relacion entre los resultados de las firmas y la competitividad del sistema productivo nacional. 


\section{Introduction}

Une physionomie nouvelle des PMI se dégage de nombreux travaux (Buigues, Ilzhoritz et Lebrun, 1991 ; Commission des CE, 1993 ; Deutsche Gesellschaft für Mittelstandsberatung, 1993 ; Ministère de l'Industrie, 1991 et 1992 ; Porter, 1990) qui mettent principalement en exergue la corrélation des enjeux internationaux des entreprises industrielles avec leurs efforts technologiques, les nécessaires arbitrages des dirigeants pour tendre vers la compétitivité globale et l'importance croissante des modes d'organisation pour canaliser les forces en vue d'atteindre leurs objectifs. Ces études contribuent à montrer combien les entreprises de taille moyenne sont essentielles au développement d'un tissu industriel concurrentiel.

Il leur est reconnu, en effet, de façon classique, une forte contribution à l'économie, une meilleure résistance aux crises, une plus grande aptitude à s'adapter aux changements, une capacité d'innovation élevée facilitant une régénération du tissu économique, une importante sensibilité aux besoins du marché et une intégration substantielle au milieu local de leur implantation. Cependant, il est aussi habituel de leur attribuer quelques handicaps dont voici les principaux : la difficulté à recruter et à conserver du personnel de haut niveau, la faiblesse de leurs fonds propres cumulée avec un coût de l'endettement supérieur à celui des grandes entreprises, une formation à la gestion très limitée des dirigeants, une ou des dépendances dangereuses (envers les hommes clés, les établissements financiers, les fournisseurs ou les clients), une localisation hasardeuse le plus souvent et une fragilité inversement proportionnelle à leur taille.

Toutefois, à l'instar des grandes entreprises, les dirigeants des PMI commencent à être sensibilisés à la nécessité d'entreprendre une réflexion stratégique rigoureuse au sein de leur organisation. Pour cela, ils acceptent souvent de se faire aider par des consultants, en dépit d'abus encore fréquents sur le plan de leurs facturations pour des résultats parfois décevants.

Cette ouverture nouvelle est imposée par les nécessités

- d'accroître encore le rythme de l'innovation (innover plus vite et plus fréquemment) ;

- de s'ouvrir sur les marchés extérieurs ;

- de s'ancrer plus fermement dans l'environnement local pour bénéficier de la solidarité de proximité, des externalités et des services proposés aux entreprises;

- de s'intégrer et de synchroniser les différentes fonctions pour tendre vers plus de cohérence interne. 
Au moins en Europe (Commission des CE, 1993) ${ }^{1}$, le premier résultat notable de ces prises de conscience pour les PMI, est, comme on le constate dans le cas des grandes entreprises, qu'elles accroissent leurs investissements immatériels ${ }^{2}$.

Comme chacun sait, au sein de l'Union européenne, la France et l'Allemagne sont deux puissances industrielles bénéficiant d'un réseau de PMI très développé sur lequel nous allons plus particulièrement porter notre attention. Après un bref exposé des choix méthodologiques opérés et des sources d'information utilisées, nous analyserons les comportements stratégiques et les modes de gestion des entreprises industrielles de taille moyenne françaises et allemandes afin de dégager leurs dissemblances et leurs similitudes. Puis, nous tenterons d'expliquer les causes structurelles des écarts de performance entre entreprises françaises et entreprises allemandes. Enfin, en conclusion, nous mettrons en évidence la nécessité pour les deux pays - et la France surtout de réarticuler les performances des entreprises et la compétitivité du système productif national avec les quelques idées qu'il est possible de formuler à partir de l'ensemble de l'analyse.

\section{Choix méthodologiques et origine de l'information exploitée}

Dans cette partie seront abordées successivement les caractéristiques méthodologiques de la recherche, puis les sources de l'information utilisée.

\subsection{Caractéristiques méthodologiques}

La méthodologie retenue repose essentiellement sur les principaux éléments suivants.

1. Au sein des pays composant l'Union européenne, il n'existe pas de définition commune de la PME. Dans les travaux de la Commission, il fut par conséquent décidé de classer les PME en fonction du nombre d'employés et d'en distinguer trois catégories : les micro-entreprises (de 0 à 9 salariés); les petites entreprises (de 10 à 99 salariés); les moyennes entreprises (de 100 à 499 salariés).

2. Ainsi, en France, les PMI réalisent $9,2 \%$ des dépenses intérieures de recherche, en 1992 , contre $5,6 \%$ en 1981 , et elles emploient $13,5 \%$ des chercheurs de l'industrie contre $8 \%$, onze ans auparavant. Deux indicateurs propres à l'ensemble des PMI étayent cette progression : le nombre de chercheurs par rapport aux effectifs est passé de $0,13 \%$ en 1981 à $0,33 \%$ en 1991 et la part des dépenses intérieures de recherche dans la valeur ajoutée a plus que doublé (de $0,8 \%$ à $1,7 \%$ ) au cours de la même période («Les chiffres clés des PMI », Ministère de l'Industrie, 1995); voir également sur ce sujet, Guellec et Zaidman, 1991 ; Magnier et Zaidman, 1991. 
Tout d'abord, il convient de noter l'extériorité du terrain observé par rapport aux chercheurs, du fait de l'exploitation d'informations issues d'enquêtes conduites par des spécialistes des deux pays concernés. Les données analysées n'ont donc pas été collectées et traitées par nous-mêmes, ce qui exigera quelques précisions sur les conditions de réalisation de ce travail par leurs auteurs. Ensuite, le choix de l'induction a été fait en raison de la priorité donnée au terrain, constitué d'observations instantanées relativement statiques, pour dégager diverses tendances. Enfin, les informations quasi exhaustives à notre disposition ont fait l'objet d'un traitement essentiellement qualitatif en vue d'atteindre une visée descriptive, analytique et explicative des comportements stratégiques des PMI françaises et allemandes.

En conséquence, les intérêts des choix méthodologiques faits pour répondre à la problématique développée reposent sur les éléments suivants :

- le bénéfice d'une source d'information très complète des deux côtés du Rhin, difficilement envisageable pour des chercheurs isolés (voir caractéristiques des études ci-dessous);

- l'apport d'une réflexion supplémentaire par rapport aux chiffres provenant des enquêtes conduites dans les deux pays comparés ;

- le rapprochement des deux nations leaders de l'Union européenne par rapport à leur tissu de PMI et à leurs spécificités respectives.

Le souci de comprendre et d'expliquer ce qui se passe en Allemagne, pays souvent cité comme référence en Europe, et en France au sein des PMI, oblige à trouver une base de données récentes, relativement exhaustives ou représentatives de cette population d'entreprises chez ces deux membres de l'Union européenne.

Notre choix s'est porté sur les enquêtes des pouvoirs publics allemands et français, car cette source d'information est officielle, fiable, complète, objective et rigoureuse sur le plan scientifique. C'est donc cette grande valeur informative qui a notamment prévalu dans notre décision finale. Les bases de données retenues sur les PMI sont toutefois largement complétées dans notre travail par un apport explicatif et analytique. Nous avons émis quelques réflexions en fin d'étude qui pourraient laisser croire à un positionnement prescriptif ou normatif. Il n'en est rien : notre conclusion consiste en quelques idées générales pouvant éventuellement être détaillées dans le débat difficile d'aujourd'hui se rapportant à la performance et à la conservation des activités des PMI sur le territoire national. Il ne s'agit donc pas de conclusions définitives, mais uniquement de pistes de réflexion destinées à alimenter une discussion qui est loin d'être close. 


\section{2. Éléments d'explication des enquêtes PMI exploitées}

L'étude PMI 93 s'appuie sur l'analyse des réponses de 500 dirigeants français et 500 dirigeants allemands constituant un échantillon représentatif d'entreprises dans chacun des deux pays. Pour l'Allemagne comme pour la France, l'échantillon reproduit les poids relatifs des entreprises industrielles dans les trois strates de taille suivantes : de 100 à 199 salariés, de 200 à 499 et de 500 à 2000 , et dans les neuf grands secteurs industriels que représentent les industries agroalimentaires, les minerais et matériaux, la chimie-pharmacie, la fonderie et le travail des métaux, la construction mécanique, la construction électrique, les transports terrestres et navals, le textile, l'habillement et le cuir, les industries diverses. Afin de ne pas exclure les éléments provenant de l'industrie en pleine mutation de la partie orientale de l'Allemagne, les réponses d'un miniéchantillon de 26 entreprises localisées dans les nouveaux Länder sont également présentées en annexe.

Pour garantir la représentativité de la population enquêtée et tenir compte des différences de comportements des dirigeants (les dirigeants allemands souhaitant un contact direct avec les enquêteurs), le questionnaire a été envoyé par voie postale à l'ensemble des entreprises industrielles françaises de 100 à 2000 salariés $^{3}$, et a été administré par enquêteurs lors d'entretiens face à face en Allemagne auprès des dirigeants du même type de firmes.

Plus précisément, en France, l'échantillon représente 8,3\% des 6150 entreprises de 100 à 2000 personnes. Ce taux est en phase avec la représentativité des effectifs $(8,8 \%)$, du chiffre d'affaires $(9,5 \%)$ et de la valeur ajoutée $(8,7 \%)$. En Allemagne, l'échantillon se compose de $5 \%$ des 9950 entreprises de 100 à 2000 salariés connues dans les anciens Länder. Au sein des 30000 entreprises industrielles de 20 à 2000 salariés du système productif français, les entités de l'étude PMI 93 représentent environ 6000 firmes soit $20 \%$ en nombre d'entreprises. En termes quantitatifs, les populations françaises et allemandes sont très différentes puisque pour une entreprise française dans cette tranche d'effectif, on dénombre 1,7 entreprise en Allemagne. Dans les 32000 entreprises industrielles ouest-allemandes de 20 à 2000 salariés, les 9950 entreprises allemandes de PMI 93 représentent $31 \%$ du nombre d'entreprises.

3. Nous devrions plus justement évoquer les entreprises à taille humaine plus que les PMI en retenant cette population de sociétés pouvant atteindre 2000 personnes. Toutefois, nous conserverons la terminologie des responsables des enquêtes tout en gardant à l'esprit cette importante remarque. 
Les autres spécificités de l'étude PMI 93 reposent sur la mise en œuvre des méthodes d'enquête suivantes.

Tout d'abord, un questionnaire long et très complet a été conçu, dépassant les moyens possibles à mobiliser pour un chercheur isolé, voire une équipe peu structurée. Outil d'autodiagnostic pour le dirigeant qui le remplit, le questionnaire comprend environ 900 questions simples. Il aborde des thèmes multiples allant des enjeux stratégiques (produits, clients, marchés, organisation commerciale, concurrents, dynamique concurrentielle, alliances stratégiques et bancaires, etc.) aux priorités de gestion et aux choix d'investissement (ressources technologiques et innovation, management de la production et choix d'investissements, organisation interne, système d'information et système de gestion, ressources humaines, environnement institutionnel et financement). Ensuite, l'analyse est fondée sur l'exploitation des réponses de 500 dirigeants français et 500 dirigeants allemands de l'ex-RFA et de 26 dirigeants opérant dans les nouveaux Länder de l'Est. Enfin, l'enquête s'est déroulée en décembre 1992, janvier et février 1993 dans les deux pays et a pris en considération les habitudes des dirigeants des deux pays en matière de collecte de données.

Ainsi, l'envoi a été effectué par voie postale en France. Les retours ont été suivis au jour le jour et des relances téléphoniques ont été pratiquées, permettant d'assurer l'exploitation finale de 500 réponses représentatives du tissu global. En Allemagne, les dirigeants ont été rencontrés personnellement par les enquêteurs. L'échantillon final est, lui aussi, représentatif de l'ensemble du tissu allemand des entreprises de 100 à 2000 salariés. Après relecture de l'ensemble des questionnaires, les informations ont été saisies informatiquement et les matrices de données obtenues ont fait l'objet d'un contrôle. Dans certains cas, le retour au questionnaire, voire au dirigeant ou à l'entreprise, a été effectué, assurant une grande fiabilité de même qu'une très grande qualité aux résultats publiés.

\section{Dissemblances et similitudes des PMI françaises et allemandes}

Si nous comparons la France et l'Allemagne ${ }^{4}$, nous pouvons dégager, d'une part, de grandes disparités des structures industrielles des deux pays et, d'autre part, de fortes convergences en matière de management stratégique.

4. Nous nous appuierons, dans ce deuxième point, essentiellement sur les travaux suivants:

- le rapport «PMI 93 » du ministère de l'Industrie et du Commissariat général du plan (1994) dont il a été question dans la première partie ;

- le rapport «Dynamiques du système productif français : de la création de richesses » (Levet et Colletis, 1995). 


\subsection{Disparités structurelles des deux tissus industriels}

D'une façon générale, les deux tissus de PMI sont structurellement différents. Si l'on s'en tient à quelques variables disponibles dans les deux enquêtes qui ne reprennent malheureusement pas en totalité - faute d'informations pertinentes présentes - toutes les dimensions judicieuses listées en introduction pour qualifier les nouvelles PMI (enjeux internationaux, efforts technologiques, comportements des dirigeants et types d'organisation), le tissu industriel allemand des PMI (20 à 499) est plus dense que celui de la France (voir tableau ci-après) pour tous les indicateurs quantitatifs habituellement retenus en la matière (nombre d'entreprises, effectifs employés, chiffre d'affaires réalisé, valeur ajoutée dégagée).

Figure 1

Les PMI en France et en Allemagne

(Industrie hors agro-alimentaire et armement, 1990)

\begin{tabular}{|c|c|c|c|c|c|c|}
\hline & \multicolumn{4}{|c|}{ Entreprises employant } & \multirow{3}{*}{$\begin{array}{l}\text { Total de } \\
\text { l'industrie }\end{array}$} \\
\hline & & \multicolumn{3}{|c|}{ PMI } & \multirow{2}{*}{$\begin{array}{l}500 \\
\text { personnes } \\
\text { et plus }\end{array}$} & \\
\hline & & $\begin{array}{c}20 \text { à } 99 \\
\text { personnes }\end{array}$ & $\begin{array}{l}100 \text { à } 499 \\
\text { personnes }\end{array}$ & $\begin{array}{c}\text { Total } \\
\text { des PMI }\end{array}$ & & \\
\hline Nombre d'entreprises & $\begin{array}{l}\text { France } \\
\text { Allemagne }\end{array}$ & $\begin{array}{l}18517 \\
21544\end{array}$ & $\begin{array}{l}4109 \\
7951\end{array}$ & $\begin{array}{l}22626 \\
29495\end{array}$ & $\begin{array}{r}871 \\
1903\end{array}$ & $\begin{array}{l}23497 \\
31398\end{array}$ \\
\hline Effectifs employés & $\begin{array}{l}\text { France } \\
\text { Allemagne }\end{array}$ & $\begin{array}{l}791382 \\
992965\end{array}$ & $\begin{array}{r}847131 \\
1640214\end{array}$ & $\begin{array}{l}1638513 \\
2633179\end{array}$ & $\begin{array}{l}2537711 \\
4116294\end{array}$ & $\begin{array}{l}3176224 \\
6749473\end{array}$ \\
\hline Chiffre d'affaires H.T. & $\begin{array}{l}\text { France } \\
\text { Allemagne }\end{array}$ & $\begin{array}{l}521 \\
575\end{array}$ & $\begin{array}{r}661 \\
1100\end{array}$ & $\begin{array}{l}1182 \\
1675\end{array}$ & $\begin{array}{l}1622 \\
3363\end{array}$ & $\begin{array}{l}2804 \\
5038\end{array}$ \\
\hline Valeur ajoutée * & France & 176 & 209 & 385 & 486 & 871 \\
\hline & Allemagne & 232 & 417 & 649 & 1209 & 1858 \\
\hline
\end{tabular}

* Valeur ajoutée brute au coût des facteurs.

Unités : CAHT et valeur ajoutée - milliards de francs 1990.

Champs : entreprises de 20 à 499 personnes.

Source : SESSI, Ministère de l'Industrie, France, «Les chiffres-clés des PMI », 1995.

L'écart est encore beaucoup plus marqué en prenant les entreprises industrielles moyennes ou qualifiées à taille humaine dans notre pays (jusqu'à 2000 personnes) puisqu' on en recense 9950 en Allemagne contre 6150 en France mobilisant de 100 à 2000 salariés.

La structure sectorielle des deux tissus révèle que $27 \%$ du «Mittelstand » allemand appartiennent à la mécanique, contre $12 \%$ pour la France, ce qui 
n'est pas à proprement parler surprenant lorsqu'on connaît la réputation et le dynamisme de nos voisins dans cette activité. Les entreprises allemandes de taille moyenne ont une ancienneté supérieure aux entreprises françaises de dimension équivalente 5 . Enfin, le degré d'autonomie des entreprises de moyenne dimension est plus élevé en Allemagne qu'en France ${ }^{6}$. La moindre «qualité » de la relation grandes entreprises-PMI, observée en France, est souvent considérée comme un des éléments négatifs de son tissu industriel. Les groupes, selon Lafay (1994),

[...] s'efforcent d'externaliser de multiples opérations en fonction des conditions de coût, au risque parfois de perdre leur savoir-faire. L'élément-clé est la nature des relations qui s'établissent au sein du tissu industriel national: ou bien, des grands groupes industriels établissent des liens durables de partenariat avec leurs soustraitants, qu'ils aident à se développer - exerçant alors un puissant effet d'entrâinement sur l'ensemble du tissu industriel - ou bien, au contraire, les groupes privilégient pour leurs achats la rentabilité immédiate, cherchant à s'approvisionner aux meilleurs prix en France ou à l'étranger. Si les conditions macro-économiques ne sont pas favorables, ils risquent alors d'exercer une pression excessive sur leurs soustraitants français et de se tourner, de plus en plus, vers des sous-traitants étrangers.

Cette remarque met en évidence un point important de la base de données utilisée. En effet figurent au sein des PMI retenues à la fois les vraies et les fausses. Ces dernières sont toutes les filiales de groupes, qu'elles soient contrôlées par le biais de participations financières suffisantes (elles sont alors prises en considération par les statistiques) ou par le marché du fait d'une dépendance économique de la plus petite structure (plus délicates à observer hors des entités économiques elles-mêmes). Ces fausses PMI peuvent être la conséquence d'une stratégie de réorganisation interne avec souci de continuer à conserver le contrôle du management de la nouvelle société ou d'une stratégie de PMIsation purement juridique fondée sur la répulsion sans contrôle financier, mais avec une forte dépendance de la nouvelle organisation créée (Nunes, 1991). Dans ce dernier cas, la sous-traitance peut être fondée sur un état d'esprit de coopération, et l'on parlera d'impartition «noble» ou, au contraire, elle peut être fondée sur un désir du client d'obtenir au meilleur compte ses achats et il s'agira d'impartition «sauvage» (Paturel et Nunes, 1987).

Toutes ces situations sont présentes aussi bien en Allemagne qu'en France. Toutefois, au moins deux types d'explication peuvent être avancés

5. Dans l'échantillon «PMI 93 », l'ancienneté moyenne allemande est de 58 ans contre 40 ans pour les entreprises moyennes françaises.

6. L'appartenance à un groupe concerne $31 \%$ du Mittelstand et $51 \%$ des entreprises françaises de taille moyenne (PMI 93). 
pour rendre compte des différences de comportement des groupes industriels à l'égard des structures PMI sous-traitantes de part et d'autre du Rhin :

- les fournisseurs et les équipementiers allemands ne souffrent pas, dans leur ensemble, des mêmes handicaps que leurs homologues français. En particulier, globalement, la formation professionnelle allemande est mieux adaptée aux besoins des entreprises ; il est vrai aussi que celles-ci font vivre de façon plus opportune cette activité outre-Rhin qu'en France. Comme les grandes entreprises, les PMI allemandes peuvent ainsi s'appuyer sur une main-d'œuvre ouvrière qualifiée, ce qui leur permet de proposer une offre spécifique et non uniquement des productions à faibles coûts, obtenues par l'usage intensif d'un personnel sans qualification.

- la propension des firmes allemandes à coopérer entre elles, à tisser des relations de longue durée constitue un phénomène économique, mais aussi culturel inscrit dans le champ des représentations des acteurs de ce pays, alors que c'est beaucoup moins vrai en France.

\section{2. Émergence de convergences fortes}

Vision stratégique, axes de développement, organisation de l'entreprise constituent trois convergences fortes des tissus allemands et français des PMI et, plus spécialement, des firmes de taille moyenne ${ }^{7}$.

\subsection{Vision stratégique : vers une stratégie de la multicompétence}

Les entreprises françaises et allemandes ont la même perception de leur champ concurrentiel, c'est-à-dire qu'elles dégagent les mêmes conclusions de l'analyse structurelle de leur activité. Elles ont aussi la même façon d'envisager leurs relations avec les autres compétiteurs. Aussi, les stratégies développées sont communes et sont des stratégies d'expansion horizontale avec accent mis sur la spécialisation et une plus ou moins forte différenciation. L'étude PMI 93 classe les stratégies de ces entreprises, en fonction du produit et du mode de production, en quatre catégories : la domination par les coûts, la différenciation des produits, la spécialisation et le simple suivi (d'un leader) par imitation. Les résultats obtenus montrent que les PMI françaises et celles du Mittelstand déclarent, chacune de son côté, à $40 \%$ suivre une stratégie de spécialisation

7. C'est ce qui apparaît en particulier à travers l'enquête réalisée dans le cadre du rapport PMI 93 que nous utilisons pour donner un contenu à ces trois points. 
de produit. Vient ensuite la stratégie de différenciation (par rapport à la concurrence). Le leadership par les coûts n'est que très minoritairement recherché tandis qu'une stratégie de suiveur intéresse moins de $2 \%$ du Mittelstand et $9 \%$ des PMI.

On remarquera que la spécialisation n'est pas incompatible avec la domination par les coûts, ni avec la différenciation d'une part, et que les stratégies génériques de Porter sont loin, dans le temps et l'espace, d'être elles-mêmes en opposition : il est aisément constaté sur le terrain que les firmes (y compris les PMI) optent pour une stratégie à un moment donné et complètent le premier choix par une autre stratégie générique lors d'une période ultérieure 8 .

La compétitivité «hors prix »-privilégiée en particulier par les entreprises allemandes et souvent considérée comme une stratégie gagnante pour les PMI -, qui est supposée à elle seule garantir l'obtention d'une position significative et durable sur les marchés les plus importants, semble atteindre ses limites. Le maintien de la rentabilité passe désormais par des retours sur investissements plus rapides qui exigent progressivement à la fois une stratégie de différenciation des produits (par la technologie) et une stratégie de maittrise importante des coûts. Plus de $40 \%$ des entreprises de l'échantillon PMI 93 ( $41 \%$ en France et $44 \%$ en Allemagne) s'engagent dans cette voie médiane qui était encore considérée comme dangereuse il y a quelque 15 ans.

Ces entreprises cherchent donc à dépasser la traditionnelle opposition entre compétitivité prix et compétitivité «hors prix» en s'appuyant sur un développement privilégiant, en outre, les relations avec les clients et les fournisseurs, la maîtrise des canaux de distribution, le développement technologique (de produits et de processus). C'est le second point de convergence entre les entreprises françaises et allemandes. Il s'agit encore d'une application des stratégies génériques au sein de ce type d'entreprises des deux côtés du Rhin, donnant toute leur pertinence à leur contenu et à leur valeur sur le terrain, y compris au niveau des PMI. Cela ne pourrait surprendre le spécialiste pour lequel toute stratégie de développement de l'entreprise, quelle que soit sa taille, se positionne nécessairement sur le tableau synoptique récemment élaboré des manœuvres stratégiques génériques (Paturel, 1997).

8. Sur le débat déjà bien nourri autour de la possibilité de se situer à un moment donné sur la voie médiane, il est possible de consulter : Phillips et al., 1983; Dess et Davis, 1984 ; White, 1986 ; Miller et Friesen, 1986 ; Wright, 1987 ; Wright et Parsinia, 1988 ; Hill, 1988; Kim et Lim, 1988 ; Jones et Butler, 1988; Speed, 1989 ; Denis et Quon, 1991 ; Miller, 1992 ; Jeanblanc, 1992 ; Parker et Helms, 1992 ; Nayyar, 1993 ; Corten et Will, 1993 ; Reistperger et al., 1993 ; Miller et Dess, 1993. 


\subsection{Axes de développement: une combinaison nouvelle}

PMI 93 fait ressortir quatre orientations dont les entreprises cherchent à assurer la combinaison, à savoir les développements commercial et technologique, le souci encore timide d'assurer la transmission de l'entreprise et la prise en compte des contraintes de l'environnement (au sens écologique du terme).

- La priorité accordée à la fonction commerciale consiste à établir des liens plus étroits avec les clients ( $80 \%$ de l'échantillon) et les fournisseurs (35\%). Cette prise de conscience de l'intérêt du partenariat (encore insuffisante avec les fournisseurs) constitue un tournant important dans l'esprit des dirigeants des PMI et une véritable force pour l'avenir.

- L'impératif technologique revient, pour $70 \%$ de l'échantillon (pourcentage identique en France et en Allemagne), à se fixer comme objectif de créer de nouveaux produits tous les trois ans. Manifestement, les dirigeants de PMI montrent, pour une forte majorité d'entre eux aujourd'hui, une grande lucidité par rapport aux conditions de maintien dans les affaires et aux choix stratégiques à opérer afin de répondre à la nouvelle donne de l'environnement national et international.

- Par contre, Allemands et Français n'ont pas encore majoritairement pris conscience de l'importance de la préparation de leur succession au sein des PMI qu'ils dirigent. En effet, $43 \%$ des entreprises françaises et $40 \%$ du Mittelstand n'ont pas résolu leur problème de succession. Cela laisse ces organisations dans une grande dépendance envers cet homme clé que représente souvent le dirigeant d'entreprise de petite et moyenne dimension, aussi bien pour sa succession financière que managériale. La conséquence de ces résultats est que le risque successoral (Paturel et Derrouch, 1987) est manifestement très fort dans la majorité des PMI françaises et allemandes, et la probabilité de voir disparaître de nombreuses organisations productives (avec les emplois qu'elles ont créés), en cas de difficultés survenant au dirigeant, demeure très élevée. Des actions sont à mener d'urgence pour sensibiliser davantage les dirigeants à ce problème et des mesures sont à prendre par les pouvoirs publics pour aider à résoudre les difficultés liées à cette situation.

Pour ce qui concerne l'échantillon PMI 93, d'une façon générale, les entreprises allemandes existent depuis plus longtemps que les entreprises françaises (58 ans contre 40 ans d'ancienneté moyenne), et $28 \%$ de ces dernières ont été créées après 1973 contre seulement $7 \%$ pour les sociétés allemandes.

- Enfin, la prise en compte de l'environnement a émergé, en quelques années, comme l'une des dimensions à venir de la compétitivité des entreprises. Ainsi, $66 \%$ des entreprises françaises et $80 \%$ des entreprises 
allemandes envisagent d'avoir à s'adapter aux pressions écologiques dans les trois prochaines années, action citée plus fréquemment que les préoccupations de diversification ou d'intégration. On notera le plus fort pourcentage obtenu en Allemagne qui s'explique par une présence beaucoup plus soutenue du mouvement écologiste dans ce pays par rapport à celui vivotant en France.

Cette adaptation n'est pas vécue négativement comme un facteur de limitation du développement futur des PMI par la moitié de ces mêmes entreprises ( $33 \%$ en France et $44 \%$ en Allemagne). Ces résultats dénotent une évolution très sensible des attitudes. Les PMI ne sont même que $3 \%$ en France et $9 \%$ en Allemagne à estimer que le coût de la protection de l'environnement pourrait motiver une délocalisation de leur production, ce qui laisse supposer que ce coût est acceptable et supportable pour une grande majorité de ces entreprises.

Tout compte fait, ce sont les entreprises qui pratiquent la nouvelle stratégie gagnante combinant différenciation et maîtrise des coûts qui sont les plus nombreuses $(78 \%)$ à intégrer les contraintes environnementales dans leur réflexion stratégique (approche de l'environnement global) et leur développement.

\subsection{Organisation de l'entreprise : autour de la flexibilité}

PMI 93 précise les leviers conduisant à la flexibilité généralisée recherchée par les entreprises industrielles de taille moyenne. L'externalisation d'une partie importante de la valeur ajoutée par l'achat de travaux sous-traités - qui a pour conséquence, par PMIsation économique (Nunes, 1991), de transformer des charges fixes en charges variables et de diminuer le seuil de rentabilité de l'entreprise impartitrice, toutes choses égales par ailleurs - caractérise davantage les entreprises allemandes (30\% contre $14 \%$ pour les entreprises françaises). La coordination des relations avec les clients et les fournisseurs s'effectue par échanges de données informatisées pour les tissus français et allemands, même si les premiers se centrent plutôt sur la synchronisation de fonctions de production et les seconds sur d'autres informations (transmission des commandes par exemple). La circulation de l'information par la réduction des niveaux hiérarchiques est un mouvement déjà ancien en Allemagne, mais qui progresse bien en France. Enfin, dans le cadre de l'élaboration et du développement de nouveaux produits, le décloisonnement des services est une forte préoccupation pouvant tendre à dynamiser le processus d'innovation.

Ainsi, d'une façon générale, la maîtrise des technologies de l'information devient un paramètre déterminant de l'évolution organisationnelle des entreprises. Les deux tiers des entreprises interrogées estiment d'ailleurs achevée l'informatisation de l'ensemble de leurs fonctions. 
À côté de ces convergences fortes, l'enquête PMI 93 confirme une différence de fond qui réside dans la façon de mailler les tissus industriels. En simplifiant, on peut constater que les entreprises françaises de taille moyenne ont tendance à se limiter à la fabrication des produits et à la recherche d'une certaine maîtrise technologique, alors que les entreprises allemandes de même dimension, pour une part significative d'entre elles, rentrent dans une nouvelle étape consistant à se définir à partir du savoir-concevoir, du savoir-faire et du savoir-vendre (notion de compétences, voire de métier?).

Cette différence renvoie aux évolutions historiques respectives des tissus allemands et français. En effet, depuis les années 1980, le tissu industriel français est passé d'une logique de production dominante (où la mise au point de produits est pratiquement déconnectée de l'étude du marché) à une logique industrielle (prise en compte des clients et de l'importance de la technologie). Au cours de la même période, le Mittelstand passe d'une logique industrielle à une logique de réseaux (voir les travaux notamment de Laurent, 1993, Chauchefoin, 1994, Poulin, Montreuil et Gauvin, 1994). L'innovation est conçue comme un processus interne et externe à l'entreprise et comme le résultat d'un maillage de réseaux avec les clients, les fournisseurs, les centres de recherche et les universités.

Pour résumer cette partie de la recherche, on peut dire que les modes de représentation varient d'un pays à l'autre. L'approche est plutôt individualiste en France, où la coopération et la concurrence sont en opposition. L'approche est concertée en Allemagne où coopération et concurrence se combinent. Diverses dimensions caractérisent la physionomie nouvelle des PMI. Ainsi, pour ce qui concerne les modes socio-organisationnels, les PMI françaises mettent prioritairement l'accent sur les innovations organisationnelles internes visant à corriger des lourdeurs structurelles, alors que les PMI allemandes développent une gestion intégrative des sous-traitants par liaisons informatiques. De même, le maillage clients-fournisseurs est plus dense outre-Rhin qu'en France, en particulier par le recours à la coordination informatisée des moyens de production qui intègre le réseau de sous-traitants. Ou encore, en matière de gestion des ressources humaines, dans les PMI françaises, des actions dans tous les azimuts, voire disparates, sont conduites au coup par coup sans véritable stratégie sur le long terme combinant mobilité interne et plans de formations professionnelles. Dans les PMI allemandes, la gestion paraît plus maîtrisée, plus concertée et davantage fondée sur la recherche de l'amélioration des savoirfaire des salariés. Primes de motivation et accords sur la durée du travail sont des leviers bien intégrés.

Par conséquent, il s'agit maintenant d'identifier les obstacles que les PMI françaises doivent franchir pour combler leur écart stratégique globalement défavorable par rapport aux entreprises allemandes. 


\section{Les obstacles à la mutation au sein des PMI françaises}

On peut relever deux barrières à la mutation des PMI françaises (et surtout des entreprises moyennes étant donné notre source d'information), également causes structurelles des écarts de performances avec leurs homologues allemands (Levet et Colletis, 1995) :

- le choix d'une stratégie de compétitivité indifférenciée et réductrice,

- une capacité insuffisante à coordonner ou à coopérer.

\subsection{Une stratégie de compétitivité indifférenciée et réductrice}

Redressement apparent du solde du commerce extérieur industriel ${ }^{9}$, productivité du travail supérieure à celle de nombreux autres pays industriels ${ }^{10}$, compétitivité-prix acquise sạns dévaluation, taux d'autofinancement élevé ${ }^{11}$, tels sont les indicateurs exprimant un redressement apparent significatif de la compétitivité des entreprises françaises.

Ce diagnostic peut sans doute se vérifier au niveau micro-économique auprès de telle ou telle entreprise ou groupe aujourd'hui en bonne santé alors que sa situation était fragile, il y a encore quelques années. Sur un plan plus global, il convient également de souligner le remarquable désendettement

9. Solde du commerce extérieur français, balance des biens industriels.

$\begin{array}{lrrrr} & 1991 & 1992 & 1993 & 1994 \\ \text { Produits manufacturés } & -35,5 & 3,8 & 43,1 & 52,5 \\ \text { Total des biens } & -84,3 & -21,7 & 32,2 & 32,1\end{array}$

Données en milliards de francs.

Source : INSEE, L'économie française, Paris, Livre de poche, 1995.

10. En dollars courants, la France est au deuxième rang après le Japon. Un emploi français produit, en moyenne, $55370 \$$ de valeur ajoutée contre $61710 \$$ pour un emploi japonais, $54710 \$$ pour un emploi américain et $52360 \$$ pour un emploi allemand.

En dollars ppa courants (parité des pouvoirs d'achats), l'effet taux de change est supprimé et l'on se rapproche des conditions comparatives acceptables de productivité du travail. La productivité du travail français est encore très élevée (au deuxième rang après les États-Unis) et celle du Japon diminue du fait de la forte surévaluation du yen par rapport au dollar et aux autres monnaies en fin de période.

11. Le taux d'autofinancement des entreprises non financières françaises est passé de $60 \%$ en 1980 à $77 \%$ en $1985,92 \%$ en $1990,103 \%$ en 1993, $140 \%$ en 1994 (Source : INSEE, 1995). 
des firmes françaises ${ }^{12}$ alors que ces dernières souffraient d'une indéniable contrainte de financement de leurs investissements au début des années 1980.

Si un certain nombre de «signaux » ou d'indicateurs peuvent ainsi laisser penser que les entreprises françaises disposent aujourd'hui d'une meilleure santé, notamment financière, qu'il y a dix ans, il persiste certains dysfonctionnements qu'il convient à présent d'exposer de manière synthétique en explicitant ce que nous entendons par «stratégie de compétitivité indifférenciée et réductrice».

À l'aube des années 1980 et au regard d'une situation financière particulièrement dégradée, les entreprises françaises ont moins privilégié la recherche durable de profits satisfaisants que leur simple survie à court terme. Pour une part non négligeable d'entre elles, le risque était celui de la cessation d'activité. Rappelons qu'on est passé, en France, de 15864 faillites en 1979 à 26395 en 1986 et à plus de 73000 aujourd'hui, soit, avec une base 100 en 1979, à près de 460 en ce moment (INSEE). Qu'il s'agisse des PME fragilisées par la longueur de la crise et la difficulté d'accès aux financements privés et publics, ou des grands groupes dont la situation financière et industrielle dégradée est sans doute à l'origine du vaste mouvement de nationalisation qui a servi de contexte à d'importantes rectifications de frontières et de légitimation à des transferts financiers considérables, on peut affirmer que la situation de nombre d'entreprises françaises était des plus critiques. Aux difficultés de stratégie industrielle souvent liées (grands groupes) à une croissance externe, à une internationalisation ou une diversification mal préparées et incorrectement maîtrisées (Boissin, 1994), a répondu le piège de l'endettement (les taux d'intérêt réels étant positifs).

Pouvant difficilement remettre en cause leur stratégie industrielle dans un délai court (problème de l'irréversibilité limitée de la décision stratégique), les entreprises françaises ont cherché à se désendetter tout en tirant un plus grand avantage de leur compétitivité en coût. La désindexation des salaires par rapport aux prix, la baisse de la part des salaires dans la valeur ajoutée ${ }^{13}$, la réduction substantielle du nombre d'emplois ${ }^{14}$ ont ainsi permis le rétablissement spectaculaire de la part des profits dans cette même valeur ajoutée.

12. En pourcentage de la valeur ajoutée, l'endettement brut des entreprises françaises est de $13,2 \%$ en 1989, 9,5\% en 1991, 6,7\% en 1992, 0,8\% en 1993 (Source : Banque de France, 1994).

13. De $77 \%$ en 1983, elle est passée à $71 \%$ en 1985 , puis à $68,5 \%$ en 1992 .

14. Sur la période 1980-1992, la France a perdu 1,85\% d'emplois industriels par an en moyenne. 
L'accroissement du chômage a fini par peser sur les performances des entreprises, davantage à cause de l'insuffisant dynamisme de la demande qu'en raison de l'accroissement des charges sociales liées au nombre croissant d'actifs ayant perdu leur emploi ${ }^{15}$. En d'autres termes, tant au niveau macroéconomique qu'au niveau micro-économique, la manière la plus immédiate de réduire les coûts de production a consisté à contracter la masse salariale par la suppression d'emplois et la diminution du coût salarial à l'unité produite. Si, dans les années 1980, un effort certain a été engagé - illustré par l'industrie automobile - pour mettre en œuvre une stratégie de compétitivité fondée également sur l'innovation, la manœuvre indifférenciée suivie a été contrecarrée par un certain nombre de faits.

Tout d'abord, des stratégies de même type engagées plus tôt par les firmes étrangères concurrentes (dans des secteurs comme la sidérurgie, le textile et l'habillement, la mécanique ou la chimie). Ensuite, le retour d'une compétition par les coûts et les prix émanant cette fois de pays caractérisés par un très faible coût horaire du travail, voire des capacités technologiques croissantes (l'Italie et plus généralement les pays de l'Europe du Sud ainsi que certains pays d'Asie du Sud-Est). Enfin, la difficulté de passer d'une stratégie d'innovation inspirée d'un schéma linéaire et intégré (de la recherche-développement au marché) à une stratégie fondée sur la combinaison de compétences internes et externes à l'entreprise (coopérations technologiques) et des boucles de rétroaction, comme le recommande le modèle de réflexion stratégique traditionnel.

En effet, ce constat fréquent provient des obstacles culturels et informationnels liés au choix d'une stratégie en cohérence avec la zone de compatibilité issue des diagnostics stratégiques internes (que peut faire l'entreprise avec ses compétences distinctives actuelles et potentielles ?) et externes (que pourrait-elle envisager d'harmonieux avec les aspirations de ses dirigeants ?). Ces difficultés de natures diverses privent les PMI et le tissu industriel notamment d'un mode d'organisation en réseau efficace dont on connaît les fondements et les implications sur la dépendance (Vallerand, 1996), la flexibilité en finance et en production, la spécialisation de chaque membre dans ses domaines de prédilection.

Ainsi, aux effets pervers liés à la façon dont était recherchée l'amélioration de la compétitivité-coût (ralentissement de la demande) se sont ajoutées les difficultés à élargir les termes de la compétitivité par le développement de stratégies visant l'innovation, rendant la stratégie choisie trop réductrice.

15. M. Vilbenoît, président de la CFE-CGC, a rappelé que pour un salaire au plafond de la Sécurité sociale, les cotisations des salariés ont augmenté de $67 \%$ en quinze ans contre $3 \%$ pour les cotisations patronales (Le Monde du 28 janvier 1995). 
En dépit du rétablissement du taux d'autofinancement (souligné précédemment), l'action conjuguée de taux d'intérêt élevés, d'une incertitude forte sur les évolutions à moyen et à long terme de la demande, et de structures de bilans portant encore la trace de "survaleurs », a eu comme conséquence d'élever la norme de rentabilité des investissements. Mais il conviendrait de ne pas confondre des stratégies fondées sur un comportement d'attente ou le refus d'engagements irréversibles dans un contexte d'incertitude, et des stratégies de spéculation définies comme n'ayant d'autres finalités que le profit qu'elles peuvent engendrer (Bourguinat, 1994). Il est clair, cependant, que la distinction entre ces deux stratégies n'est pas aisée et que, surtout, la première peut déboucher sur la seconde, si l'incertitude se prolonge.

\subsection{Une capacité insuffisante à coordonner ou à coopérer}

Le thème du partenariat est sans doute un des thèmes ayant été les plus traités tant dans les discours politiques que dans la littérature économique et de gestion depuis les années 1980 notamment (Barreyre et Bouche, 1982; Garrette et Dussauge, 1995). Plutôt qu'un raisonnement en termes de partenariat, lequel peut laisser supposer l'absence de conflits ou de rapports de force, notre analyse utilisera les termes de coopération et de coordination des activités économiques.

Les principales questions qui se posent sont donc les suivantes :

- Pourquoi les formes de coordination gagnent-elles aujourd'hui en importance?

- Pourquoi les entreprises françaises semblent-elles avoir plus de difficultés que leurs concurrentes à coopérer?

$\mathrm{Si}$, aujourd'hui, de nombreuses entreprises préfèrent faire faire plutôt que faire elles-mêmes, c'est qu'elles y trouvent un avantage économique certain. Ce dernier tient fondamentalement dans les termes suivants : minimisation de l'ensemble des coûts de production et des coûts de transaction, partage des coûts ou des risques lorsque ceux de la croissance interne ou externe sont trop élevés, complémentarité des compétences ou des activités, recherche et création de poche de stabilité au sein d'un environnement concurrentiel fort.

Le domaine particulier de l'innovation technologique illustre bien cette perspective. Deux entreprises qui décident de coopérer, de développer des projets de recherche ou d'innovation communs le font pour réduire ou partager les coûts et les risques qui y sont liés. Souvent, leur coopération engage des compétences complémentaires au service d'un même objectif. On parlera, si le but n'est pas d'aboutir à un produit commun aux contractants, de partenariats verticaux ou d'accords intersectoriels lorsque les entreprises en relation ne sont 
pas concurrentes, et d'alliances de cointégration dans le cas contraire (Garrette et Dussauge, 1995).

Une quinzaine de recherches de bon niveau (et au moins autant d'ouvrages) ont été réalisées dans le cadre de travaux doctoraux en France depuis cinq ans sur le thème de l'internalisation ou de l'externalisation d'activité. Il ne paraît pas utile de reprendre ici leur teneur (et à plus forte raison de privilégier l'un ou l'autre d'entre eux), car ils se fondent tous sur les mêmes bases théoriques et l'originalité ne provient souvent que du terrain d'application ou de l'activité étudiée. Toutefois, cet engouement pour la recherche dans ce domaine confirme que la question est d'actualité, mobilise les énergies et n'est peut-être pas encore épuisée.

La seconde interrogation, plus ardue à traiter, consiste à rendre compte du faisceau de motifs qui permet de comprendre pourquoi les entreprises françaises semblent avoir plus de mal à coopérer que les entreprises concurrentes étrangères (Coriat et Taddei, 1993 ; Boyer, 1993). En termes de méthode, il convient de démontrer ce déficit spécifique aux entreprises françaises - et plus particulièrement aux PMI, très individualistes - et de l'expliquer. Les comparaisons internationales relatives à la propension des firmes à coopérer sont peu nombreuses et fragiles ${ }^{16}$. En particulier, la nature de la coopération est souvent mal cernée, portant sur des produits, des services, des dispositifs techniques, des connaissances, des personnes, voire du capital.

À défaut de mesurer directement la propension des firmes à coopérer, il est possible de l'évaluer ou de la repérer de diverses façons. Il est envisageable de comparer des ratios d'efficience du type investissement / valeur ajoutée ou dépenses de $\mathrm{RD} /$ brevets. À titre d'illustration, il a pu être montré, pour un même secteur (la sidérurgie), que l'industrie allemande était plus économe en investissements que l'industrie française (Marger, 1979), c'est-à-dire qu'une unité monétaire d'investissement créait plus de valeur ajoutée en Allemagne qu'en France. À partir de l'observation de ratios d'efficience différents selon les pays, on peut faire l'hypothèse (qui serait à conforter) qu'une des raisons d'un ratio d'efficience inférieur est le défaut de coordination des acteurs ${ }^{17}$.

La difficulté des entreprises françaises à coopérer - en dépit d'un « taux d'externalisation », semble-t-il, plus élevé que celui observé dans les entreprises

16. Se reporter cependant aux études FAST de la Communauté européenne (Direction générale-XII).

17. Il convient d'évoquer, ici, le caractère central des accords verticaux entre producteurs et distributeurs (Rey, 1994). Ces accords sont plus particulièrement appréhendés dans la littérature sur les « systèmes nationaux d'innovation ». 
de divers pays, notamment en Allemagne ${ }^{18}$ - peut faire l'objet d'un certain nombre de suppositions. Leur dénominateur commun est l'accent mis sur la question des représentations. La coopération ne supprime pas la compétition, ni le marché. En revanche, elle infléchit, voire met entre parenthèses, les exigences et les contraintes immédiates de la concurrence. La perspective de coopération, si elle s'inscrit en fonction de la quête de réalisation d'un avantage économique "objectif», suppose une disposition culturelle particulière susceptible d'être résumée par au moins deux notions clés : la confiance et la réciprocité. Ces deux notions clés ont été investies plutôt par la sociologie que par l'économie ou la gestion. Cependant, des travaux récents, à la frontière des deux disciplines, mettent l'accent sur l'importance des phénomènes d'apprentissage organisationnel (c'est-à-dire le transfert au partenaire des savoir-faire difficiles à formuler et à formaliser) et soulignent le rôle fondamental de la convergence des anticipations dans le temps de la stratégie des différents acteurs (Eymard-Duvernay, 1994 ; Midler, 1994 ; Guilhon, 1994 ; Ruffieux et Robin, 1995).

En se fondant sur l'idée de l'existence de systèmes exprimant simultanément des dispositifs institutionnels de promotion de l'innovation spécifiques à chaque pays (Lundvall, 1992 ; Chouraqui, 1994) et un système de représentation (normes, règles et valeurs) [Hodgson, 1988] propre à chaque culture nationale, il est possible de produire des images relativement différenciées des " modèles » nationaux types, modèles à la fois culturels et politiques, sociaux et économiques (Albert, 1991). Le modèle allemand, entre autres caractéristiques, semble devoir correspondre à une référence forte à la compétition et à la coopération (culture du consensus, communauté de destin) [Hofstede, 1991; Gigon, 1994]. Le modèle français, en revanche, aurait plutôt tendance à opposer compétition et coopération, et à affirmer le primat de l'individu sur le groupe ou la communauté d'intérêt.

18. En effet, une représentation erronée de la réalité de l'industrie allemande consiste à penser qu'elle est moins intégrée que l'industrie française. C'est en fait l'inverse qui est vérifié. En moyenne, le taux d'intégration (VA/CA) de l'industrie allemande est supérieur à celui observé en France $(35,1 \%$ contre $31,5 \%)$ (Ministère de l'Industrie, SESSI, 1991). Les activités commerciales n'expliquent pas à elles seules ce résultat. Les firmes françaises font plus appel que leurs concurrents allemands à des fournisseurs pour produire, comme le met en évidence pour la France le ratio VA/Production propre qui élimine « l'effet négoce » $(34,6 \%$ contre 39,1\%) (Ministère de l'Industrie, SESSI, 1991). Aussi, les entreprises allemandes préfèrent-elles davantage que les firmes françaises contrôler de l'intérieur l'ensemble du processus de production (de la conception à l'assemblage). Toutefois, l'intégration plus ou moins élevée de la production (ou son inverse, l'externalisation) n'est pas nécessairement un indicateur de la propension des entreprises à travailler ensemble. 
Cette caractérisation est discutable puisqu'elle porte sur des représentations supposées et $s$ 'applique de manière générale à des acteurs qui sont insérés dans des cultures nationales spécifiques. Ces derniers, qui œuvrent au sein de contextes particuliers, disposent d'une autonomie individuelle au point qu'il est possible d'affirmer, d'une part, que les perceptions constituent un «moteur» de leurs actions et, d'autre part, que les pratiques coopératives dépendent de leurs représentations des relations existant avec l'environnement (Jovène, 1995).

Le passage, inachevé en France, vers la firme-réseau et le réseau de firmes (voir les travaux déjà cités) qu'illustre, par exemple, l'étude PMI 93, pourrait ainsi être expliqué par un modèle culturel moins favorable à une telle évolution ou conception de l'organisation que ne le seraient les modèles japonais ou allemand au centre desquels se trouveraient des valeurs de référence telles que l'esprit d'équipe et le consensus.

Un troisième obstacle peut éventuellement être identifié. En effet, une exploitation sous-estimée et peu efficace d'un important potentiel territorial de coopération est à relever dans notre pays. De plus, l'analyse en termes d'insertion internationale et de globalisation des stratégies trouve un corollaire indispensable dès lors que l'on raisonne sur la base de l'architecture spatiale d'un système productif ouvert et que l'on admet que la globalisation signifie aussi une différenciation accrue de l'espace mondial (voir les travaux de Vernon, 1966 ; Hamel et Prahalad, 1985 ; Porter, 1986 ; Prahalad et Doz, 1987 ; Bartlett et Goschal, 1989 ; et la recherche internationale en cours coordonnée par Atamer, 1995, ESC Lyon).

C'est de la correspondance (ou de l'absence de correspondance) entre les stratégies des firmes et la nature des dynamiques territoriales que naissent (ou ne naissent pas) des « rencontres productives». Celles-ci peuvent être de différentes natures, mais sont susceptibles d'être ramenées à deux cas de figure. Le premier est interprétable en termes d'allocation optimale de ressources, le second, en termes de création de ressources nouvelles.

- Le premier type de rencontre productive est centré sur une logique de coûts : la firme localise ses activités, c'est-à-dire sa production, ses investissements et ses emplois, dans les pays où les coûts des facteurs sont les moins élevés. Les politiques publiques d'aménagement du territoire ont consisté, pour l'essentiel, à corriger les effets de la concentration spatiale (polarisation) explicables par une logique de moindre coût ou de disponibilité des facteurs ${ }^{19}$.

19. Ainsi, la prime à l'aménagement du territoire visait-elle à réduire (temporairement) le coût du travail dans les zones prioritaires. 
- Le second type échappe en partie à une logique exprimée exclusivement en termes de coûts. En effet, si l'on raisonne uniquement sur ce plan, et quel que soit le niveau des aides publiques, il y a fort à craindre que les firmes globales, par définition mobiles, parviennent toujours à découvrir de meilleurs lieux d'implantation que ceux de l'Europe et de la France en particulier (du fait d'un système de veille environnementale développé notamment), même si cette dernière est probablement un des pays européens les plus attractifs ${ }^{20}$. C'est précisément la comparaison internationale des coûts de production qui a fondé les craintes de fuite des emplois récemment exprimées en France (Arthuis, 1993 ; Naigeon, 1994).

Cette seconde logique concerne donc la relation entre la firme et le territoire dans une perspective de flexibilité dynamique et de création de ressources (Zimmermann, 1995). Si les firmes, présentes dans un environnement incertain, développent une aversion marquée pour les engagements irréversibles, elles peuvent néanmoins investir parfois durablement dans un processus de construction territoriale, dès lors qu'une telle option leur permet de résoudre des problèmes productifs inédits, c'est-à-dire sortant d'une logique d'équilibre ou de routine pour entrer dans une logique de constitution de ressources nouvelles.

Les études menées dans ce domaine commencent à bien cerner le sujet pour les entreprises de grande taille, mais restent, lorsqu'elles existent, de portée extrêmement limitée pour les PMI. Nous ne pouvons donc aller sérieusement plus avant dans la démonstration, sauf peut-être pour rappeler que les PMI allemandes sont probablement mieux ancrées dans leurs territoires que les PMI françaises et bénéficient ainsi de coopérations de complémentarités (Ministère de l'Industrie, Commissariat général du plan, 1994).

\section{Conclusion}

\section{Performances des entreprises et tissu industriel national: une articulation à réinventer}

Le fait nouveau est que, désormais, en France notamment, les entreprises les plus grandes ${ }^{21}$ peuvent prétendre dissocier leur devenir de celui de l'économie nationale. L'accélération des investissements directs à l'étranger (facilitée par

20. En 1992, la France a été le deuxième pays d'accueil des flux d'investissements directs, derrière la Grande-Bretagne et avant le Bénélux et l'Espagne. Voir en particulier: «Investir en France : un espace attractif », Commissariat général du plan, La Documentation Française, 1992.

21. Mais aussi quelques PMI (voir les travaux menés actuellement à Montpellier sur la globalisation au sein de la petite entreprise par O. Torrès, ERFI, 1997). 
le pouvoir d'achat élevé du franc) [Paturel, 1991, 1992, 1993 et 1994], la délocalisation possible d'une partie significative des activités de production, y compris dans des activités de haute technologie (électronique), et la croissance de la part des ventes réalisées dans d'autres pays ont fini par produire une situation telle que le lien entre les performances des entreprises et le devenir du système productif a eu tendance à se détendre. C'est cette relation qu'il convient de densifier, de fortifier d'une façon probablement plus urgente qu'en Allemagne.

La globalisation des stratégies et le nomadisme des firmes mettent aujourd'hui les territoires étrangers et nationaux en concurrence. L'enjeu est ainsi à la fois de rendre l'espace national attractif et moins dépendant des fluctuations de l'environnement international et de décisions prises par des opérateurs dont les objectifs sont autonomes. À terme, l'objectif est de recouvrer une meilleure maîtrise des ressorts internes du développement.

La relation firmes-territoire national est entendue non comme une relation de contrainte réciproque, mais comme une relation de flexibilité dynamique. Le maintien et le développement d'activités productives localisées sur le territoire national sont synonyme d'avantages économiques pour les firmes qui y participent. Ces avantages ne doivent pas être acquis sur la base - le plus souvent fragile et provisoire - d'un accès artificiellement peu onéreux aux facteurs de production. L'accroissement très sensible des emplois « subventionnés 22 » dans le secteur marchand, s'il peut être justifié par les contraintes de court terme imposées par un niveau de chômage élevé, ne tient pas lieu de stratégie.

Les entreprises sont invitées à participer à la construction économique du territoire national et à maintenir ou à créer des emplois en France sans que cela constitue pour elles un objectif à atteindre comme tel, mais parce qu'il s'agit d'un résultat (non intentionnel) d'une stratégie visant des objectifs propres (profit, part de marché, survie). Cette stratégie est, selon cette perspective, synonyme de réalisation d'objectifs macro-économiques par définition extérieurs à toute firme, fût-elle de grande dimension. D'ailleurs, n'est-ce pas là également un élément de la responsabilité sociale et sociétale de l'entreprise ? Cette responsabilité est à développer par les collectivités publiques notamment territoriales lors de leur définition de moyens d'actions en faveur des PMI. De plus, la formation des dirigeants des entreprises à taille humaine devrait également assurer une sensibilisation à une responsabilité sociale et sociétale plus

22. D'après le premier bilan de la politique de l'emploi du ministère du Travail, le secteur privé (emplois marchands) a drainé environ un million d'emplois subventionnés au cours de l'année 1994 («Le travail dans vingt ans », Commissariat général du plan, 1995). 
élevée par rapport à ce qu'elle propose aujourd'hui, un peu comme l'enseignement a évolué en matière d'éthique ces dernières années. Ce nouvel effort assurerait la prise en considération, d'une part, lors de l'étude de l'environnement global de la firme, de l'importance du facteur sociétal et, d'autre part, au moment de l'analyse de l'entreprise, de l'introduction de la variable sociale et, donc, de la responsabilité de la PMI envers ses salariés et leur famille, et envers son espace géographique et économique d'influence. La réflexion stratégique s'en trouverait complétée et élargie, tandis que la firme deviendrait un acteur à l'action plus volontaire en matière aussi bien sociale que sociétale, avant même que le législateur la contraigne trop dans ces domaines, comme il le fait très souvent.

\section{Bibliographie}

Albert, M. (1991), Capitalisme contre capitalisme, Paris, Éditions du Seuil, Collection L'histoire immédiate.

ARTHUIS, J. (1993), Les délocalisations industrielles, Paris, Document du Sénat français.

BARREYRE, P.-Y. et M. Bouche (1982), « Pour une compétitivité fondée sur la solidarité entre les entreprises », Paris, Revue Française de Gestion, no 37, p. 8-17.

Bartlett, C. A. et S. Goschal (1989), Managing Across Borders. The Transnational Solution, Boston, Harvard Business School Press.

BoIssin, J.-P. (1994), Construction d'un cadre d'analyse des déterminants de la performance dans les opérations de diversification par acquisition, Thèse en sciences de gestion, Grenoble, Université de Grenoble II, 421 pages.

Bourguinat, H. (1994), «Renégocier Bretton Woods ? La spéculation internationale comme trouble-fête », Communication au colloque EFIQ-GDR-CNRS, sur la négociation commerciale et financière internationale, Sophia-Antipolis, juin.

BOYER, R. (1993), «How to promote cooperation within conflicting and divided societies?", Paris, CEPREMAP (Centre d'études prospectives d'économie mathématique appliquée à la planification), nº 93-23, décembre.

Buigues, P., F. ILZHORITZ et J.-F. LEBRUN (1991), L'impact sectoriel du marché intérieur sur l'industrie : enjeux pour les États membres, Paris, Economica.

CHAUCHEFOIN, P. (1994), Les réseaux productifs : de l'organisation des activités au développement des territoires, Poitiers, Thèse en sciences économiques, Université de Poitiers, 443 pages.

Chouraqui, D. (1994), Systèmes nationaux d'innovation. Nature, fonctionnement et débats, Grenoble, Université P.-Mendès France, Institut d'études politiques et Commissariat général du plan, novembre. 
COMMISSARIAT GÉNÉRAL DU PLAN (1992), Investir en France: un espace attractif, Paris, La Documentation Française.

COMMISSARIAT GÉNÉRAL DU PLAN (1995), Le travail dans vingt ans, Paris, Éditions O. Jacob, octobre.

COMMISSION DES COMMUNAUtÉs EuRopéEnNes (1993), Panorama de l'industrie communautaire 1991-1992, Bruxelles.

CORIAT, B. et D. TADDEI (1993), Made in France. L'industrie française dans la compétition mondiale, Paris, Le Livre de Poche.

CORTEN, H. et T. WILL (1993) : «Reflections on competitive strategy and its impact on modern production concepts », International Review, vol. 33, $\mathrm{n}^{\circ} 4$.

DENIS, J. E. et T. QUON (1991), «The impact of European economic integration on outsiders generic strategies : the case of Canadian multinationals », Research in Global Strategic Management, vol. 2, p. 241-251.

DESS, G.C. et P.S. DAVIS (1984), « Porter's generic strategies as determinant of strategic group membership and organizational performance », Academy of Management Journal, vol. 27, n 3, p. 467-488.

Deutsche Gesellschaft für Mittelstandsberatung (1993), Europe PMI, Deutsche Bank.

EYMARD-DUVERNAY, F. (1994), «Coordination des échanges par l'entreprise et qualité des biens », dans Analyse économique des conventions, sous la direction de A. Orlean, Paris, Presses universitaires de France.

Garrette, B. et P. Dussauge (1995), Les stratégies d'alliance, Paris, Les Éditions d'Organisation, 283 pages.

Gigon, F. (1994), Les déterminants fondamentaux de la compétitivité. Le modèle allemand, Grenoble, Université P.-Mendès France, Commissariat général du plan, juin.

GuELleC, D. et C. ZAIDMAN C. (1991), « Recherche-Développement : un avantage à l'Allemagne », Économie et Statistique, no 246-247, p. 91-97.

GuIlhoN, B. (1994), Formation des compétences et apprentissage organisationnel, Paris, Document de travail du CEFI, $n^{0} 4$, février.

Hamel, G. et C.K. Prahalad (1985), « Do you really have a global strategy?», Harvard Business Review, juillet-août, p. 139-148.

HILL, C.W.L. (1988), « Differenciation versus low cost or differenciation and low cost : a contingency framework », Academy of Management Review, vol. 13, no 3, p. 401-412.

Hodgson, G.M. (1988), Economics and Institutions. A Manifest for a Modern Institutional Economics, Cambridge, Posity Press. 
Hofstede, G. (1991), Cultures and Organizations, Maastricht, Mc-Graw Hill.

INSEE (1995), L'économie française, Paris, Livre de poche.

Jeanblanc, P. (1992), "Modèle de gestion combiné des stratégies de coûts et de différenciation », Paris-HEC, Communication au colloque de l'AIMS.

JONES, G.R. et J.E. BUTLER (1988), «Costs, revenue and business level strategy », Academy of Management Review, vol. 13, n² 2, p. 202-213.

JovÈNE, J. (1995), Le rôle des représentations dans la compétitivité, Grenoble, Université P.-Mendès France, Institut d'études politiques, Commissariat général du plan, janvier.

KIM, W.C. et Y. LIM (1988), « Environment, generic strategies and performances in a rapidly developing country : a taxonomy approach », Academy of Management Journal, vol. $31, \mathrm{n}^{\circ} 4$, p. 802-827.

LAFAY, G. (1994), L'industrie française face aux défis de la mondialisation, Paris, Commissariat général du plan, juillet.

LAURENT, P. (1993), Les réseaux stratégiques : un essai de conceptualisation, Thèse en sciences de gestion, Lyon, Université de Lyon III, 596 pages.

LEVET, J.-L. et G. COLLETIS (1995), Dynamiques du système productiffrançais : de la création de richesses, Paris, Commissariat général du plan (document interne).

LundVAlL, B.A. (1992), National Systems of Innovation Towards a Theory of Innovation and Interactive Learning, Londres, Printer Publishers.

MAGNIER, A. et C. ZAIDMAN (1991), «Investissement : l'enjeu du redéploiement industriel », Économie et Statistique, n 246-247, p. 59-107.

MARgER, P.L. (1979), La situation et l'avenir de la sidérurgie, Paris, Conseil économique et social, Séances des 3 et 4 juillet.

MIDLER, Ch. (1994), «Évolution des règles de gestion et processus d'apprentissage», dans Analyse économique des conventions, sous la direction de A. Orlean, Paris, Presses universitaires de France.

MilleR, D. (1992), «The generic strategy trap », The Journal of Business Strategy, janvier et février, vol. $13, \mathrm{n}^{0} 1$, p. 37-41.

Miller, D. et G.G. DeSs (1993), «Assessing Porter's (1980) model in terms of its generalizability, accuracy and simplicity », Journal of Management Studies, vol. $30, n^{0} 4$, p. 553-585.

MILLER, D. et P.H. FRIESEN (1986), «Porter's (1980) generic strategies and performance : an empirical examination with American data. Part 1 : testing Porter », Organization Studies, vol. 7, n 1 , p. 37-55 ; «Part 2 : performance implications », Organization Studies, vol. 7, n 3, p. 255-261.

Ministère DE L'INDUSTRIE (1991), PMI 90, vers la compétitivité globale, Paris. 
Ministère DE L'INDUSTRIE (1992), SESSI, Paris.

MINISTÈRE DE L'INDUSTRIE (1993), MEI 92, vers la maturité de l'investissement immatériel, Paris.

Ministère DE L'INDUSTRIE (1994), PMI 93, Paris, Commissariat général du plan.

Ministère DE L'INDUSTRIE (1995), Les chiffres-clés des PMI, SESSI, Paris.

NAIGEON, G. (1994), Les délocalisations, Grenoble, Université P.-Mendès France, Institut d'études politiques, Commissariat général du plan, novembre.

NAYYAR, P.R. (1993), «On the measurement of competitive strategy : evidence from a large multiproduct U.S. firm », Academy of Management Journal, vol. 9, $\mathrm{n}^{\circ} 5$, p. $1652-1669$.

NUNES, P. (1991), Les opérations de PMIsation: pratique ou stratégie?, Thèse de doctorat en sciences de gestion, Grenoble, Université de Grenoble II, 314 pages.

PARKER, B. et M.M. HELMS (1992), «Generic strategies and firm performance in a declining industry », Management International Review, vol. 32, $\mathrm{n}^{0} 1$, p. 23-39.

Paturel, R. (1991, 1992, 1993, 1993), Chronique de la Revue d'Économie Industrielle, $\mathrm{n}^{\circ} 58,62,66$ et 70 .

PATUREL, R. (1997), Les manœuvres stratégiques génériques des entreprises, Revue Sciences de Gestion, $\mathrm{n}^{\circ} 23,24$ pages.

Paturel, R. et B. Derrouch (1987), «Les implications du risque sur les stratégies de croissance de l'entreprise », Les Petites Affiches, $\mathrm{n}^{0}$ 108, septembre, p. 14-23.

PATUREL, R. et P. NUNES (1987), «Les stratégies de PMIsation », Cahier de recherche 87-27 du CERAG, ESA de Grenoble, 39 pages.

Poulin, D., B. MonTREuIL et S. GAUVIN (1994), L'entreprise réseau : bâtir aujourd'hui l'organisation de demain, Montréal, Éditions Publi-Relais, 335 pages.

PHILliPS, L.W. et al. (1983), «Product quality, cost position and business performance : a test of some key hypotheses », Journal of Marketing, vol. 47, printemps, p. 26-43.

PORTER, M. (1986), «Changing patterns of international competition », California Management Review, $\mathrm{n}^{\circ}$ 28/2, p. 9-40.

PORTER, M. (1990), The Competitive Advantage of Nations, London, The MacMillan Press.

PraHalad, C.K. et Y.L. Doz (1987), The Multinational Mission, New York, The Free Press.

REISTPERGER, W.D. et al. (1993), « Product quality and cost leadership : compatible strategies?», Management International Review, vol. 33, $n^{\circ} 1$, p. 7-21.

REY, P. (1994), Commissariat général du plan, 9 décembre. 
RuffieuX, B. et S. RoBIN (1995), «La théorie des jeux face à l'économie expérimentale: le cas de la confiance pure », Grenoble, IREPD-IPI/ENSGI, février.

SHARP, B. (1991), «Competitive marketing strategy : Porter revisited », Marketing Intelligence and Planning, vol. 9, $\mathrm{n}^{\circ} 1$, p. 4-10.

SPEED, R. (1989), «Oh Mr Porter! A re-appraisal of competitive strategy », Marketing Intelligence and Planning, vol. 7, $\mathrm{n}^{\mathrm{os}} 5 / 6$, p. 8-11.

TORRÈs, O. (1997), Les stratégies de globalisation des petites entreprises, Thèse de doctorat en sciences de gestion, Montpellier, Université de Montpellier I.

VALLERAND, J. (1996), Essai de modélisation du passage à l'Organisation-réseau, Thèse de doctorat en sciences de gestion, Grenoble, Université de Grenoble II, 280 pages.

VERNON, R. (1966), «International investment and international trade in the product cycle », Quarterly Journal of Economics, n 87/2.

VILbEnoît, M. (1995), Journal Le Monde du 28 janvier.

WhITE, R.E. (1986), « Generic business strategy, organizational context and performance : an empirical investigation », Strategic Management Journal, vol. 1, $\mathrm{n}^{\mathrm{o}} 1$, p. 217-231.

Wright, P. (1987), «A refinement of Porter's strategies», Strategic Management Journal, vol. 8, n 1 , p. 93-101.

Wright, P. et A. PARSINIA (1988), « Porter's synthesis of generic business strategies : a critique », Industrial Management, vol. 30, n 3, p. 20-23.

ZIMMERMANN, J.B. (1995), L'ancrage territorial des activités industrielles et technologiques, Paris, Convention d'étude du Commissariat général du plan, Service du développement technologique et industriel, $n^{0} 23-94$, juillet. 